\title{
Evaluation von Empowerment - Perspektiven und Konzepte von Gesundheitsförderern. Ergebnisse einer qualitativen Studie in Australien
}

\section{Evaluation of Empowerment: Health Promotion Practitioners' Perceptions and Concepts. Results of a Qualitative Study in Australia}

\author{
Autoren \\ J. Loss ${ }^{1}$, M. Wise \\ Institute \\ ${ }^{1}$ Institut für Medizinmanagement und Gesundheitswissenschaften, Universität Bayreuth \\ ${ }^{2}$ Australian Centre for Health Promotion, The University of Sydney, Australia
}

\section{Schlüsselwörter \\ - Gesundheitsförderung \\ - Empowerment \\ - Evaluation \\ - qualitative Untersuchung}

\section{Key words \\ - health promotion practice \\ - empowerment \\ - participation \\ - evaluation \\ - qualitative study}

\section{Bibliografie}

DOI 10.1055/s-0028-1103260

Gesundheitswesen 2008;

70: 755-763

(c) Georg Thieme Verlag KG

Stuttgart · New York

ISSN 0941-3790

\section{Korrespondenzadresse}

Priv.-Doz. Dr. med. Julika Loss Institut für Medizinmanagement und Gesundheitswissenschaften

Universität Bayreuth

95440 Bayreuth

julika.loss@uni-bayreuth.de

\section{Zusammenfassung \\ $\nabla$}

Hintergrund: Empowerment ist ein Kernkonzept der WHO-Vision von Gesundheitsförderung. Die Evaluation von Empowerment erweist sich aber als schwierig. Bei der Entwicklung angemessener Studiendesigns, Indikatoren und Methoden für die Messung und Bewertung von Empowerment sollten die Erfahrungen, Bedenken und alltäglichen Rahmenbedingungen von Praktikern der Gesundheitsförderung berücksichtigt werden.

Methoden: Es wurden semistandardisierte Interviews mit 17 erfahrenen Experten der gemeindenahen Gesundheitsförderung im Bundesstaat New South Wales, Australien, durchgeführt. Die Interviews behandelten Nutzen und Probleme der Evaluation von Empowerment, Schlüsselindikatoren und methodische Aspekte. Die Interviewtranskripte wurden mithilfe der thematischen Content-Analyse ausgewertet. Die Ergebnisse wurden mit den Interviewpartnern auf einem Workshop diskutiert (sog. respondent validation).

Ergebnisse und Diskussion: Die Evaluation von Empowerment wird generell von den Gesundheitsförderern unterstützt. Wesentliche Bedingung hierfür ist, dass Empowerment explizit Bestandteil der Programmlogik bzw. der zugrundeliegenden Theorie ist. Die Interviews machten deutlich, wie wichtig es ist, dass das politische Umfeld die Evaluation von Empowerment befürwortet. Die oftmals fehlende Anerkennung von Empowerment als Ergebnis einer Intervention im Vergleich zu gesundheitsbezogenen Ergebnissen ist eine große Herausforderung für die Finanzierung von Evaluationen. Gemeindemitglieder sollten in den Evaluationsprozess einbezogen werden; allerdings bestand keine Einigkeit darüber, wie Repräsentativität erreicht werden kann. Die besten Methoden zur Erfassung von Empowerment sind nach Ansicht der Interview-

\section{Abstract \\ $\nabla$}

Background: Although the concepts of participation and empowerment are hallmarks of the WHO vision of health promotion, it is acknowledged that they are difficult to evaluate. Devising adequate study designs, indicators and methods for the assessment of participation and empowerment should take into account the experiences, concerns, working conditions and constraints of health promotion practitioners.

Methods: Semi-structured interviews were conducted with 17 experienced practitioners in community-based health promotion in New South Wales, Australia. The interviews covered benefits of and barriers to the evaluation of empowerment, key indicators and methodological aspects. Interview transcripts were examined using thematic content analysis.

Results and Discussion: The idea of evaluating empowerment is supported by health promotion practitioners, provided that empowerment is part of the programme logic and/or programme theory. The interviews highlighted the importance of a receptive environment for evaluation of empowerment and participation to take root. The resistence of health authorities towards empowerment indicators is a great challenge for funding of evaluations. Community members are to be included in the evaluation process, although it is not always easy to do so in a representative way and empowering approach. The best methods to capture whether empowerment has occurred in a programme are qualitative ones. The positive experiences that the interview partners made with innovative qualitative methods encourage further investment in developing new research designs. 
ten qualitative Verfahren. Die positiven Erfahrungen, die die Interviewpartner mit innovativen qualitativen Methoden gemacht haben, rechtfertigen eine Investition in die Entwicklung neuer Forschungsansätze.

\section{Einleitung \\ Die Bedeutung von Empowerment in gemeindenaher Gesundheitsförderung}

Seit der Alma-Ata-Erklärung und der Ottawa-Charter stellt Empowerment ein zentrales Konzept der WHO-Vision von Gesundheitsförderung dar [1,2], vor allem im Gemeindesetting. In der Gesundheitsförderung geht es zunehmend darum, soziale Ungleichheit von Gesundheit und Krankheit zu verringern. Auch hierfür wird Empowerment als zentraler Ansatz postuliert. Sowohl in der Forschung als auch in der Praxis der Gesundheitsförderung hat es sich allerdings als schwierig erwiesen, Empowerment zu operationalisieren und zu messen. Dadurch ergibt sich ein wachsender Bedarf, das Konzept des Empowerment klarer zu definieren, valide und zuverlässige Indikatoren zu entwickeln, und seine Rolle in der Förderung von Gesundheit und dem Abbau von sozialen Ungleichheiten zu untersuchen [3].

Empowerment umschreibt einen mehrschichtigen sozialen Prozess, durch den Individuen und Gruppen Verständnis für und Kontrolle über ihre Lebensbedingungen gewinnen. Als Folge sollten sie ihr soziales und politisches Umfeld so verändern können, dass sich ihre (gesundheitsbezogenen) Lebensumstände dadurch verbessern [4-6]. Neben einem individuellen („psychologischen“) sowie organisationsbezogenen Empowerment geht man davon aus, dass Empowerment auch auf Gemeindeebene erreicht werden kann [7]. Letzteres setzt sich aus mehreren Komponenten zusammen: Ein erhöhter Grad an individuellem Empowerment unter den Gemeindemitgliedern, die Entwicklung einer kollektiven politischen Aktivität, an der Gemeindemitglieder aktiv beteiligt sind, und das Erreichen von Entscheidungsmacht und Verfügungsgewalt über Ressourcen im Sinne der Gemeinde [3, 4, 7,8].

\section{Evaluation von Empowerment}

Zunehmend sieht sich auch die Gesundheitsförderung der Forderung nach Qualitätssicherung und -entwicklung sowie wissenschaftlicher Evaluation ausgesetzt [9]. Die Evaluation von Prozessen sowie kurz- und langfristigen Ergebnissen einer gesundheitsförderlichen Intervention kann helfen nachzuvollziehen, wer von der Intervention erreicht wird, sowie wie und warum derartige Interventionen funktionieren [10,11]. Die Ergebnishierarchie von Nutbeam illustriert die verschiedenen Ebenen und Typen von Ergebnissen, die in der Gesundheitsförderung erwartet werden könnten, z.B. „health literacy“ und sozialer Einfluss als erste Ergebnisse der Gesundheitsförderung, gesunde Lebensstile sowie effektive Gesundheitsdienste als intermediäre Gesundheitsergebnisse [12]. Erschwert wird die Evaluation dadurch, dass sich Gesundheitsförderungsprogramme in der Regel aus einer Vielzahl von Strategien und Aktivitäten zusammensetzen, die über einen längeren Zeitraum implementiert werden, und Ergebnisse auf der gesundheitlichen Ebene erst in ferner $\mathrm{Zu}$ kunft erwartet werden können.

Bislang haben sich Evaluationen von gemeindenahen Programmen im Wesentlichen auf die Messung von Veränderungen in Gesundheitsverhalten und -zustand bezogen. Aber Vieles spricht dafür, dass es zum Erreichen dieser Ziele notwendig ist, dass die
Zielgruppen der Programme auch die Kompetenzen und Fähigkeiten zum aktiven gesundheitsbezogenen Handeln haben; diese entwickeln sie am ehesten durch einen kombinierten Ansatz von Partizipation (Teilhabe) und Empowerment [13,14]. Im Rahmen der Evaluation kann es daher sinnvoll sein zu bewerten, inwieweit ein solcher Ansatz einer Gemeinde bzw. ihren Mitgliedern die Kapazitäten vermitteln konnte, mit denen sie ihr gesundheitsbezogenes Verhalten sowie Determinanten dieses Verhaltens beeinflussen können $[4,8,14]$.

Im internationalen Raum wurden verschiedene Variablen und Indikatoren zur Evaluation von Empowerment vorgeschlagen [15]. Diese Indikatoren beinhalten subjektive Dimensionen, wie die wahrgenommene Wissens- und Fähigkeitsentwicklung, Selbstwertgefühl, eingeschätzte Kompetenzen und Selbstwirksamkeit, Bereitschaft zur Beteiligung an kollektiven Aktivitäten oder das wahrgenommene Zusammengehörigkeitsgefühl in der Gemeinde [4,5,16-18]. Außerdem werden tatsächlich erreichte Leistungen und Verbesserungen in Umwelt- und Lebensbedingungen als Variablen vorgeschlagen, z.B. die Entwicklung neuer oder im Sinne der Gemeindebedürfnisse veränderter Programme, Strategien und Praktiken oder die vergrößerte Verfügungsgewalt über Ressourcen [7,19]. In diesem Zusammenhang wurden auch einige Befragungsinstrumente für Projektleiter bzw. Gemeindemitglieder vorgestellt. Einige wenige Studien haben qualitative Ansätze, z. B. Tiefeninterviews, als Erhebungsmethode eingesetzt $[4,16,20]$. Viele dieser Methoden sind jedoch dafür kritisiert worden, dass sie das vielschichtige Konzept von Empowerment nicht ausreichend erfassen [4,21].

Eine Reihe von Doppeldeutigkeiten und Unklarheiten erschwert die Messung und Bewertung von Empowerment. Dazu zählt insbesondere die Tatsache, dass Empowerment schwer von anderen Konzepten wie Partizipation und Capacity Building abzugrenzen ist [22]. Zudem ist umstritten, ob diese Konzepte als eigenständige Ergebnisse eines gesundheitsförderlichen Programms verstanden werden können, oder als beitragende Faktoren im Sinne von Prozessvariablen $[4,5,23]$. In ihrer Übersichtsarbeit zur Wirksamkeit von Empowerment weist Nina Wallerstein darauf hin, dass eine Investition in die Evaluationsmethoden und in die Überarbeitung von Messinstrumenten dringend benötigt wird [14].

Neben offenen theoretischen bzw. methodologischen Fragen kommt hinzu, dass bislang wenig praktische Erfahrungen zur Evaluation von Empowerment in der Literatur beschrieben sind [8]. Obwohl sich die Gesundheitsförderung seit Langem dem Konzept des Empowerment verpflichtet fühlt, gibt es nur wenig Angaben dazu, wie die Bewertung von Empowerment von Praktikern der Gesundheitsförderung wahrgenommen wird. Möchte man untersuchen, inwiefern Empowerment für die Effektivität von Gesundheitsförderung entscheidend ist, wird es notwendig werden, Evaluationsstudien in gemeindenahen Programmen zu implementieren. Hierfür ist es sinnvoll, die fördernden und hemmenden Faktoren, die sich bei der Evaluation von Empowerment in der Praxis ergeben (können), zu verstehen. Die Sicht von Projektleitern und -mitarbeitern in der Gesundheitsförderung zu diesem Thema und zum Aufbau geeigneter Evaluationsstudien ist dazu ein erster wichtiger Schritt.

\section{Evaluation von Empowerment in Australien}

Die Studie wurde in Australien durchgeführt. Grund hierfür war, dass verschiedene australische Bundesstaaten, v.a. New South Wales, sehr detaillierte und systematische Strategiepapiere oder Rahmenprogramme für Gesundheitsförderung erarbeitet haben 
$[24,25]$. Zudem ist das Einbeziehen der Bürger in Entscheidungen zur Gemeindeentwicklung in Australien besonders stark in Politik und Praxis verankert [26-28] und kann als wesentlich weiter entwickelt gelten als hierzulande. Bezeichnend ist auch, dass in Australien die Evaluation als unverzichtbarer Bestandteil gesundheitsförderlicher Programme gilt. Bereits vor Jahren wurden systematische Kataloge von Indikatoren entwickelt, z.B. zum Bereich verschiedener Ebenen der Kapazitätsbildung [24]. Auch beschäftigten sich viele australische Studien speziell mit methodischen Fragen der Evaluation einschließlich der Evaluation von Empowerment [29-32]. Aufgrund dieser Rahmenbedingungen besteht bei australischen Praktikern der Gesundheitsförderung eine besondere Kompetenz sowie eine ausgeprägte Erfahrung auf dem Gebiet der Evaluation von gemeindenaher Gesundheitsförderung, insbesondere der Evaluation von Empowerment. Diese können auch für Deutschland hilfreich und handlungsleitend sein.

Die Ziele dieser Studie waren, die Perspektiven von australischen Gesundheitsförderern zu folgenden Aspekten zu erheben:

- Nutzen von und Schwierigkeiten bei der Evaluation von Empowerment in gemeindenahen Gesundheitsförderungsprogrammen,

- Indikatoren und Messeinheiten für Empowerment und

- Studiendesign und Erhebungsmethoden für die Evaluation von Empowerment.

\section{Methoden}

$\nabla$

Es wurden semistandardisierte, face-to-face Interviews mit 17 Experten der gemeindenahen Gesundheitsförderung im Bundesstaat New South Wales, Australien, im Zeitraum von September bis November 2005 durchgeführt. Gezielte und SchneeballRekrutierung wurden zur Gewinnung der Interviewpartner eingesetzt. Die Interviewpartner arbeiteten in verantwortlichen Positionen in verschiedenen Area Health Services (vergleichbar etwa deutschen Gesundheitsämtern), im Gesundheitsministerium von New South Wales, oder an der University of Sydney und der University of New South Wales. Akademische Interviewpartner wurden nur dann eingeschlossen, wenn sie ausgeprägte praktische Erfahrungen in der gemeindenahen Gesundheitsförderung hatten. Die Rekrutierung wurde fortgesetzt, bis ein Sättigungsgrad erreicht war. Drei Gesundheitsförderungsexperten wurden darüber hinaus vorab im Sinne von Probeinterviews befragt und kommentierten anschließend den Interviewleitfaden. Diese drei Interviews wurden in die Analyse mit aufgenommen, da der Interviewleitfaden nur unwesentlich verändert wurde. Keiner der angefragten Teilnehmer hatte Bedenken hinsichtlich der Teilnahme am Interview oder zur Aufzeichnung. Um Vertraulichkeit der Angaben zu gewährleisten, waren nur die Autoren in Transkription und Analyse und der Interviews involviert. Die Aufbereitung der Daten ermöglicht dabei keine Identifikation individueller Interviewteilnehmer.

\section{Datenerhebung}

Die Interviews bezogen sich auf (1) Nutzen von und Hemmnisse für die Aufnahme von Empowerment in die Evaluation eines Programmes zur gemeindenahen Gesundheitsförderung, (2) Schlüsselindikatoren für Empowerment und (3) Vorschläge zu methodischen Aspekten für die Messung und Bewertung von Empowerment (Studiendesign, Datenquellen, Datenerhebungsmethode).
Darüber hinaus behandelten die Interviews auch die Evaluation von Partizipation sowie Definitionen von Empowerment and Partizipation, Nutzen und Grenzen von Empowerment und Partizipation in der Praxis; die Ergebnisse dieses Teils der Interviews werden anderswo beschrieben [33]. Eine der Autorinnen (JL) führte alle Interviews durch, von denen jedes aufgezeichnet wurde und 60-120 min dauerte.

\section{Analyse}

Die Interviewaufzeichnungen wurden wörtlich transkribiert und mithilfe der thematischen Content-Analyse [34] ausgewertet. Die Kategorien und Codes wurden identifiziert, indem ein Prozess der „grounded conceptualisation“ gewählt wurde [35]. Die Kategorien entsprachen den drei wesentlichen Dimensionen der Interviews, die in Themen und Unterthemen weiter diversifiziert wurden. Die Transkripte wurden vor und nach dem Codieren wiederholt gelesen, um sicherzustellen, dass die Daten korrekt kategorisiert wurden. Um die Validität der Ergebnisse zu überprüfen, wurde die Auswertung der Interviews im Dezember 2005 auf einem interaktiven Workshop diskutiert, an dem 10 der 17 Interviewten teilnahmen („respondent validation“ nach Mays and Pope [36]).

\section{Ergebnisse}

Nutzen der Evaluation von Empowerment

Die meisten Interviewpartner stimmten überein, dass die Bewertung von Empowerment wichtig ist oder zumindest interessantes Potenzial hat, insbesondere als zusätzliche Möglichkeit, die Qualität einer gesundheitsförderlichen Intervention zu messen. Außerdem kann die Evaluation ein ermutigendes Feedback für die Projektleiter bedeuten, da sich erste Entwicklungen zum Empowerment früher feststellen lassen als messbare Veränderungen im Gesundheitszustand.

- „Die Leute sollten nicht enttäuscht sein, weil sie nicht ein ganzes Universum von Gesundheitsverhalten oder Gesundheitszuständen verändert haben. Ich denke, es ist wirklich wichtig, dass wir auch diese Aspekte [Empowerment and Partizipation] mitverfolgen, um sie an uns zurückzuspiegeln, anstatt zu sagen: ,Oh, ich kann nicht messen, ob ich die Sturzgefahr für die nächsten 10 Jahre verhindert habe, ich fühle mich hilflos, ich weiß gar nicht, ob mein Programm funktioniert.

- Tab. 1 gibt einen Überblick über alle in den Interviews genannten Vorteile.

Die meisten Interviewpartner sahen Empowerment (ebenso wie aktive Teilhabe) eher als Prozess- denn als Ergebnisvariable, obwohl manche der Befragten Empowerment als unabhängiges Ziel von Gesundheitsförderung werteten.

- „[Empowerment und Partizipation] sind Prozessparameter. Aber diese Dinge sagen mehr aus über den Erfolg eines Programmes, denn bei ihnen handelt es sich um Dinge, die wir einbauen müssen für die langfristige Nachhaltigkeit. Also, in einer gewissen Art und Weise sind sie so etwas wie Prozessindikatoren, aber gleichzeitig sind sie wichtige Ergebnisse für sich. ... Die aktive Teilhabe und das Empowerment dieser Menschen wären definitiv ein Erfolgsindikator für mich. Es gibt da immer diesen Bezug zur Nachhaltigkeit“.

Einige Male wurde die Äußerung gemacht, dass Empowerment ein „Ergebnis entlang des Weges“ sei.

Es wurde wiederholt darauf hingewiesen, dass Empowerment nicht routinemäßig bei jedem Programm gemessen werden 
sollte. Ob die Evaluation von Empowerment nützlich sei oder nicht, sei von der theoretischen Grundlage eines Programmes abhängig, so die Befragten. Die Evaluation von Empowerment wurde hauptsächlich dann als wichtig angesehen, wenn es auch explizit Teil der Programmlogik ist. Es mache weniger Sinn, das Konzept des Empowerment zu bewerten, wenn es sich bei der Gemeindeinitiative um ein kleines, inhaltlich stark begrenztes oder kurzfristiges Projekt oder eine reine Aufklärungskampagne handele.

- „Ich denke, wir sollten es schon machen [die Evaluation von Empowerment]. Aber...als Projektverantwortlicher möchte ich nicht, dass meine Mitarbeiter das die ganze Zeit machen, sonst bekommen sie ihre eigentliche Projektarbeit nicht getan, also, es muss schon ein gewisses Gleichgewicht da sein“.

- „Ich würde [Empowerment] nicht routinemäßig messen, also nicht als Routine. Aber wo es wichtig ist als Teil der Logik des Programmes, oder wo es Teil der Theorie von den Dingen ist, die umgesetzt und erreicht werden sollen, dann ja“.

Die Evaluation von Empowerment würde zudem für Projektleiter die Notwendigkeit betonen, dass Gemeindemitglieder aktiv an der Entscheidungsfindung und Umsetzung teilhaben, und dass auf eine erhöhte Kapazität zum gemeinsamen Handeln hingewirkt werden muss. Auch den Gemeindemitgliedern selbst kann damit verdeutlicht werden, welche Bedeutung Empowerment hat.

\section{Barrieren für die Evaluation von Empowerment}

Einige der Befragten waren überzeugt, dass ein Rahmenkonzept für eine aussagekräftige Evaluation, die zu bedeutungsvollen Ergebnissen führt, machbar ist. Andere bemerkten, dass sie eben dies für schwierig hielten. $\bullet$ Tab. 1 listet die wesentlichen Barrieren auf, die im Zusammenhang mit der Messung und Bewertung von Empowerment genannt wurden. Ein Hinderungsgrund, der häufig erwähnt wurde, war das Fehlen einer eindeutigen Definition von Empowerment.
- „Ich denke, die erste Barriere [bei der Evaluation von Empowerment] ist: Ich habe das Gefühl, ich habe keine richtige Definition dafür, und ich kann doch nicht etwas messen, das ich nicht definieren kann, ich kann nicht etwas messen, das diffus ist“.

Als weitere wesentliche Barriere erwies sich, dass die Evaluation von Empowerment von den Geldgebern bzw. Entscheidungsträgern kaum Unterstützung erfahren würde, da das Erreichen von Kompetenzen, Selbstwertgefühl und politischen Einflussmöglichkeiten in der Regel für nicht so wichtig erachtet werde wie die eindeutig gesundheitsbezogenen Veränderungen. Selbst wenn in der Theorie beschrieben ist, dass Empowerment zu einem verbesserten Gesundheitszustand beiträgt, wird es von Geldgebern und übergeordneten Gesundheitsbehörden meist noch nicht als gleichwertig zu einer Verbesserung in der Gesundheit oder als essenzielle Voraussetzung für die Gesundheitsförderung gesehen.

- „Im Gesundheitsbereich wollen die Leute Gesundheitsergebnisse“.

- „Die Gemeinde sagt: „Für mich geht es darum, dass ich mich jetzt willkommen fühle, dass ich das Gefühl habe, dass die Leute mir zuhören, und dass ich mir jetzt deswegen zutraue, Dinge anzusprechen. Das bedeutet für mich, dass ich gesünder bin, zufriedener, besser verbunden mit der Gemeinde‘. Du erklärst das dem Bürgermeister, und der Bürgermeister sagt: ,Oh, das ist ja alles gut und schön, aber - was soll ich damit anfangen?““

\section{Indikatoren für Empowerment}

Die Interviewten nannten zahlreiche Indikatoren für die Messung von Empowerment, wie in 0 Tab. 2 dargestellt. Sie bezogen sich auf subjektive Wahrnehmungen wie Selbstwertgefühl, Selbstvertrauen oder Autonomie. Objektivere Dimensionen wie Entscheidungsgewalt oder Verfügung über Ressourcen wurden ebenfalls von einigen Befragten vorgeschlagen.

- „Also, wenn man ehrlich ist, was man messen muss, ist: Was hat man in Punkto Macht und Einfluss getan. Haben sich die Macht-

Tab. 1 Vorteile und Schwierigkeiten der Evaluation von Empowerment, beschrieben durch die interviewten Gesundheitsförderer.

\section{Nutzen und Vorteile}

Die Evaluation von Empowerment kann...

- die Beteiligung der Teilnehmer würdigen

- ein ermutigendes „Feedback“ für Projektmitarbeiter bedeuten, wenn sie als ,Ergebnisse von Gesundheitsförderung k klassifiziert werden, die früher gemessen werden können als gesundheitsbezogene Ergebnisse

- das Verständnis von den Programmbestandteilen verbessern, die den Bedürfnissen der Gemeinde entsprechen (Identifizierung von ,best practice')

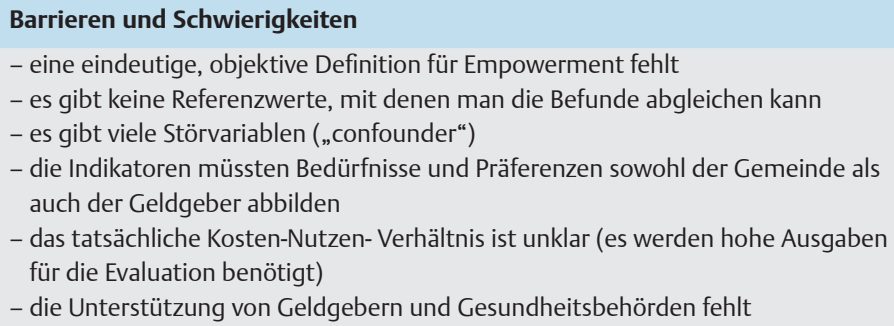

Tab. 2 Indikatoren, die für die Evaluation von Empowerment von den interviewten Gesundheitsförderungsexperten genannt wurden.
subjektive Aspekte
- Selbstwertgefühl; Sich-Wohlfühlen in seiner Haut
- Gefühl der persönlichen Autonomie
- Selbstwirksamkeit; Zuversicht, Entscheidungen treffen zu können
- Fähigkeiten, für die eigenen Interessen aktiv einzutreten („advocacy skills“)
- das Gefühl, sich Gehör verschaffen zu können
- das Gefühl, über die Ressourcen verfügen zu können
- die Fähigkeit, andere hinsichtlich z. B. Verhaltensänderungen beraten und beeinflussen zu können
objektive Aspekte
- Zugang zu gesundheitsbezogenen Leistungen und Informationen
- Verfügung über Ressourcen
- Verschiebung der Machtverhältnisse und Entscheidungsdynamiken
- veränderte politische Strategien und Regelungen
- Veränderungen im Gemeindeumfeld 
und Einflussverhältnisse verschoben? Sind die Gruppen, die jetzt Einfluss auf den Projektverlauf haben, auch diejenigen Gruppen, die tatsächlich einflussreich sein sollten? Das sind die Arten von Fragen, die wir stellen sollten, denn sonst hat man ein Projekt, das riesig im Umfang ist, riesig in der Reichweite, riesig im Budget, aber es sind immer noch diesselben fünf Leute, die das Sagen haben“.

Darüber hinaus wurde klar, dass die Indikatoren spezifisch sein müssen für a) den Programmtyp und b) den jeweiligen Gemeindekontext.

Einige Interviewpartner gaben $\mathrm{zu}$ bedenken, dass Empowerment nicht nur auf der individuellen oder Gemeinde-Ebene gemessen werden kann, sondern man auch tatsächliche Veränderungen und Auswirkungen nachverfolgen muss, z. B. auf Lebenswelten und politische Entscheidungen, denn „Empowerment nur um des Empowerments willen bedeutet rein gar nichts, bis auf dass ich mich gut über mich selber fühle, aber alles um mich herum ist immer noch das Gleiche“.

- „Bei aktiver Teilhabe und Empowerment geht es darum, ob die Menschen das GEFÜHL haben, dass ihnen zugehört wird, aber es geht auch darum, ob ihnen WIRKLICH zugehört wird“.

Die Auswirkungen von Empowerment nachzuverfolgen kann bedeuten...

- nachzuprüfen, ob konkrete Vorschläge von Gemeindemitgliedern aufgenommen wurden, z.B. nach einer Komittee-Sitzung,

- die Auswirkungen auf bestimmte Institutionen zu analysieren, z.B. Regierung, Schulen oder Kirchen und

- die Auswirkungen auf Lebenswelten zu monitoren z.B. neue politische Entscheidungen, Veränderungen in der materiellen und sozialen Umwelt.

Methodisch können Auswirkungen und Einflusswege nachvollzogen werden, indem man analysiert, welche Prozesse tatsächlich stattfinden, nachdem ein Gemeindemitglied eine Meinung geäußert hat oder einen Vorschlag in einer Arbeitsgruppensitzung gemacht hat.

- „In Bezug auf, sagen wir einmal, Fluglärm [kann man untersuchen:] Können die Gemeindemitglieder mit anderen Leuten darüber reden, bekommen sie Einfluss? Ja/nein, das kann man messen. Und haben sie etwas verändern können, haben sie die Flugrouten beeinflusst? Also hat Empowerment tatsächlich ganz konkrete Messparameter. Also, verändern sie tatsächlich politische Entscheidungen?, oder wird der Flugverkehr tatsächlich umgeleitet"?

Es wurde darauf hingewiesen, dass man unterscheiden muss zwischen der individuellen Ebene von Empowerment und der Gemeindeebene.

- „Es ist einfach wichtig, den Unterschied klar zu halten zwischen dem Einzelnen und der Gemeinde als Ganzes, oder Organisationen. Die Frage ist, bezieht sich dieses qualitative Feedback allgemeiner auf die gesamte Gemeinde, oder sind es nur ein paar Leute, die sich mit der ganzen Sache prima fühlen, und kein anderer ist davon wirklich berührt worden“.

\section{Studiendesign und Datenerhebung für die Evaluation von Empowerment}

Übergreifend über alle Interviews wurde deutlich, dass qualitative and interaktive Methoden der Datenerhebung bevorzugt werden, wenn es um die Messung von Empowerment geht. Begründet wurde dies meist damit, dass Empowerment als komplexe, subjektive und Kontext-bedingte Entität durch Fragebögen, die primär quantitative Aussagen messen, nicht ausreichend erfasst werden kann.

- „Empowerment ist ein sozial sehr vielschichtiges Konzept, und ich denke nicht, dass ein standardisierter Fragebogen das notwendigerweise entschlüsseln und erkunden kann. Nur indem du mit den Leuten sprichst, kannst du das erreichen“.

- „Wenn man es einfach nur quantitativ bestimmt, dann verliert man das, was aktive Teilhabe wirklich bedeutet“.

- „Also man kann so eine Art von Vorher-Nachher-Untersuchung machen, aber ... [in vielen Situationen] funktioniert das nicht wirklich. Also greift man zurück auf die qualitative Arbeit, denn innerhalb der Machbarkeit der meisten Projekte, ihrer Zeitrahmen, ist das die beste Evidenz, die man bekommen kann. Und das ist kein „Billigangebot“, das nichts wert ist; da ist richtige Bedeutung drin“.

- „[Evaluation von Empowerment]. Da geht es doch darum, dass die einzelnen Dinge etwas zählen - und nicht darum, Dinge zu zählen“.

Checklisten, die von den Projektmitarbeitern selber ausgefüllt werden, wurden von den meisten Interviewpartnern für die Evaluation von Empowerment abgelehnt; als Gründe wurden genannt, dass Checklisten a) zu objektiv und starr sind für das kontextuelle, teilweise subjektive Konzept von Empowerment, und b) in der Kontrolle von den Experten stehen und möglicherweise zu ihren Gunsten beeinflusst werden. Daher kann es mit diesen Checklisten eher nicht gelingen, die Dimension der Entscheidungsgewalt („power“) zu reflektieren, um die es beim Empowerment auch zentral geht.

- „Ich denke, wenn man Instrumente hat [wie Checklisten], dann erhöht man nur die Wahrscheinlichkeit, dass man am Ende ein ,leeres Ritual' durchführt. Denn solche Instrumente, die sind wie eine Liturgie“.

Die Befragten schlugen eine Vielzahl von qualitativen Methoden vor, die sich für die Evaluation von Empowerment als nützlich erwiesen haben oder als geeignet erachtet wurden ( $\bullet$ Tab. 3). Manche der vorgeschlagenen Bewertungsansätze können von den Projektmitarbeitern selbst umgesetzt werden (z.B. moderierte Selbstreflexion), aber die Mehrzahl der genannten Evalua-

Tab. 3 Vorgeschlagene qualitative Verfahren für die Evaluation von Empowerment.

\begin{tabular}{|c|c|c|}
\hline & $\begin{array}{l}\text { Datenquelle: } \\
\text { Projektteam }\end{array}$ & Gemeindemitglieder \\
\hline \multirow[t]{2}{*}{$\begin{array}{l}\text { vorgeschlagene qualitative } \\
\text { Methoden: }\end{array}$} & $\begin{array}{l}\text { - Selfreflexion der Projektmitarbeiter } \\
\text { - Reflektive Tagebücher } \\
\text { - Moderierte Gruppenreflexion }\end{array}$ & $\begin{array}{l}\text { - partizipatorische Aktionsforschung } \\
\text { - reflektive Tagebücher von Gemeindemitgliedern } \\
\text { - Story Dialogue-Methode }\end{array}$ \\
\hline & $\begin{array}{l}\text { - Dokumentation } \\
\text { - Aufzeichnung und Beschreibung der Geschehnisse } \\
\text { (zu evaluieren durch eine externe Person) } \\
\text { - Diskursanalyse der Kommunikation mit } \\
\text { Gemeindevertretern in Arbeitskreisen }\end{array}$ & $\begin{array}{l}\text { - Most Significant Change (MSC-)Methode } \\
\text { - Schauspiel } \\
\text { - Bürgerjury } \\
\text { - Photodokumentation als non-verbale Ausdrucksform }\end{array}$ \\
\hline
\end{tabular}


Tab. 4 Empfehlungen zu methodologischen Aspekten für die Evaluation von Empowerment.

\begin{tabular}{|c|c|c|}
\hline Indikator & Datenerhebung & Datenquelle \\
\hline \multicolumn{3}{|l|}{ Geldgeber/Entscheidungsträger } \\
\hline - Einsatz für und Bekenntnis zu Empowerment und Partizipation & $\begin{array}{l}\text { - Budget für Empowerment und } \\
\text { Partizipation }\end{array}$ & Finanzierungsplan \\
\hline \multicolumn{3}{|l|}{ Gesundheitsförderer, Projektteam } \\
\hline $\begin{array}{l}\text { - Einbezug der Gemeinde } \\
\text { - Einfluss der Gemeindemitglieder auf das Projekt und Verschiebung } \\
\text { von Machtverhältnissen }\end{array}$ & $\begin{array}{l}\text { - Dokumentation } \\
\text { - (moderierte) Selbstreflexion }\end{array}$ & $\begin{array}{l}\text { Projektmitarbeiter } \\
\text { - moderiert durch Experten } \\
\text { - evaluiert durch unabhängige externe } \\
\text { Person }\end{array}$ \\
\hline \multicolumn{3}{|l|}{ Gemeindemitglieder } \\
\hline \multicolumn{3}{|l|}{ Individuelle Ebene } \\
\hline \multicolumn{3}{|l|}{$\begin{array}{l}\text { - Selbstwertgefühl, Zuversicht } \\
\text { - Fähigkeit, für die eigenen Interessen einzutreten } \\
\text { - Perzeption von Einfluss }\end{array}$} \\
\hline \multicolumn{3}{|l|}{ Ebene von Gemeindeorganisationen } \\
\hline $\begin{array}{l}\text { - Kompetenzen, Kapazitäten und Durchsetzungvermögen einer } \\
\text { Gruppe }\end{array}$ & - quantitative Datenerhebung & Mitglieder der Gruppe (moderiert) \\
\hline \multicolumn{3}{|l|}{ Gemeindeebene } \\
\hline - Verantwortung für das Projekt, Führungsqualitäten & $\begin{array}{l}\text { - Dokumentation } \\
\text { - qualitative und quantitative Daten- } \\
\text { erhebung }\end{array}$ & $\begin{array}{l}\text { Projektmitarbeiter Gemeindemitglieder } \\
\text { (moderiert) }\end{array}$ \\
\hline \multicolumn{3}{|l|}{$\begin{array}{l}\text { - Veränderung der Machtverhältnisse } \\
\text { - Mobilisierung von Ressourcen }\end{array}$} \\
\hline \multicolumn{3}{|l|}{ Materielles und soziales Umfeld } \\
\hline $\begin{array}{l}\text { - veränderte politische Strategien und Regelungen } \\
\text { - Veränderungen im Gemeindeumfeld }\end{array}$ & $\begin{array}{l}\text { - Dokumentation } \\
\text { - Bezug der Veränderungen zu } \\
\text { Vorschlägen und Aktivitäten von } \\
\text { Gemeindemitgliedern }\end{array}$ & \\
\hline
\end{tabular}

tionsmethoden zielt darauf ab, diejenigen in die Datenerhebung zu involvieren, die die angestrebten Nutznießer des Programmes sind.

- „Qualitative Erhebungsmethoden. [wie z.B.] Schauspiel. sind eine sehr hilfreiche Maßnahme, denn dabei geht es um die Artikulation der Gemeinde. Die Gemeinde bekommt die Möglichkeit, sich Gehör zu verschaffen. Und was man als Gesundheitsförderer machen muss, ist, wie mit einem Lautsprecher das zu verstärken, was die Gemeinde sagt. Und natürlich ist man dann auch dazu verpflichtet, zuzuhören“.

Die Teilnehmer eines gesundheitsförderlichen Programms sollten so weit als möglich miteinbezogen werden - in die Entwicklung sowohl der Indikatoren als auch des Evaluationsdesigns.

- „Die wichtigste Eigenschaft eines solchen [Evaluations-]Ansatzes ist, dass die Teilnehmer so viel Einfluss wie möglich auf das Studiendesign haben. Denn je mehr der Ansatz zur Untersuchung durch die Projektleiter bestimmt wird, desto wahrscheinlicher wird sie zu ihren Gunsten beeinflusst, während die Untersuchung so gestaltet sein sollte, dass sie zugunsten der Teilnehmer beeinflusst wird, und die Teilnehmer die Möglichkeit haben, ihre Meinung zu sagen“.

Ein wesentliches Hemmnis für qualitative Evaluationsmethoden ist, dass die Geldgeber oder Autoritäten die eher üblichen quantitativen Ergebnisse bevorzugen.

- „Und qualitative Methoden, egal, wie gut man es macht, egal wie gut die Rationale und die Kriterien sind und die Evidenz, dass das wirklich valide und nützliche Daten sind, - es kommt dann ein ganz selbstverständliches ,Wo sind die Zahlen?‘ ... Im Gesundheitsbereich treten wir in Australien immer noch gegen das Paradigma der Evidenz-basierten Medizin an“.
Für Gesundheitsförderer besteht die Herausforderung daher darin, Wege zu finden, qualitative Ergebnisse für die Entscheidungsträger akzeptabel zu machen, z. B. indem die Ergebnisse in einer sehr präzisen, strukturierten Weise vorgestellt werden, und indem man sehr klare Konsequenzen für Planung und Umsetzung daraus ableitet. Ein alternativer Weg zur Überzeugung von Entscheidungsträgern, der häufig genannt wurde, war die Kombination von qualitativen mit quantitativen Datenerhebungen.

\section{Wer soll befragt werden?}

Es wurden verschiedene Datenquellen vorgeschlagen. Während Projektleiter interessante Einsichten haben können, sprachen sich die meisten Interviewpartner entschieden dafür aus, dass die Gemeindemitglieder in eine aussagekräftige Evaluation mit einbezogen werden müssen. Das kann passieren, indem man entweder involvierte Gemeindegruppen bzw. Gemeindesprecher befragt, oder indem man ganz normale Bürger befragt. Einige der befragten Experten bezweifelten jedoch, ob man durch das Befragen willkürlich gewählter Gemeindemitglieder signifikante Ergebnisse erhalten könnte. Zumeist wurde vorgeschlagen, mehrere Datenquellen zu verwenden.

- „Man sollte die Leute aus der Gemeinde fragen, und ich würde vielleicht auch die Projektmitarbeiter fragen; deren Ansichten werden sehr verschieden sein von dem, wie es die Gemeinde sehen wird. es würde einem eine Art Vergleich geben“.

- „So würde ich das nicht machen, ein paar zufällige Gemeindemitglieder zu fragen: ,Hast du das Gefühl, Einfluss auf das Projekt zu haben?. Ich meine, es wird dann ein paar wenige geben, die sagen: ,Ja, sehr', und die meisten Leute werden sagen: ,Nein, überhaupt nicht“". 
Wenn man die Gemeindemitglieder befragt, sollte man berücksichtigen, welche Sprache und Begriffe dabei verwandt werden. Ein wissenschaftlicher Jargon oder Ansätze von oben herab sollten ebenso vermieden werden wie der Eindruck, die Evaluation sei wichtiger als das eigentliche Projekt.

- „Offensichtlich müssen noch spezielle Techniken dazu entwickelt werden, um eine solche Befragung auch respektvoll zu machen... Es kommt darauf an, wie du an die Informationen gelangst ...in einer respektvollen Art und Weise. Denn das kann sehr schnell sehr paternalistisch sein“.

- „Der Begriff, Forschung“verunsichert die Leute total, ...sie sagen dann, ,Hä, Forschung?!؛ Also. du gehst das Ganze an in einer Art, die keine großartigen Begriffe benutzt und sich die Leute nicht irgendwie aufregen lässt über Forschung“.

- Tab. 4 stellt eine Übersicht dar über vorgeschlagene Indikatoren, Datenerhebungsmethoden und Datenquellen.

\section{Diskussion}

Die interviewten australischen Experten der Gesundheitsförderung hatten das Gefühl, dass die Evaluation von Empowerment in bestimmten gemeindebezogenen Projekten sinnvoll ist. Die genannten Vorteile und Nutzen bezogen sich dabei nicht nur auf das Projekt selbst (im Sinn von Qualitätssicherung), sondern auch auf die Gemeindemitglieder. Die offizielle Aufnahme von Empowerment als Indikator für die Evaluation würde die Bedeutung dieses Ansatzes deutlich nach außen darstellen und so sowohl für Projektleiter als auch für Gemeindemitglieder unterstreichen, dass es bei dem Projekt (auch) um die Vermittlung von Kompetenzen und die Übertragung von Einfluss geht. Auch das Evaluationsdesign und der Evaluationsprozess selber können dazu beitragen, die Entscheidungsgewalt der Gemeinde zu vergrößern. Bürgerjurys, Theatergruppen oder das Dokumentieren von Entwicklungen in Tagebüchern sind einige der Methoden, die eingesetzt werden, um sowohl den Einfluss („power“) von Bürgern zu vergrößern, als auch die Auswirkungen einer gesundheitsförderlichen Intervention zu evaluieren. Das stimmt mit dem Verständnis einiger Autoren überein, die die Überzeugung vertreten, dass der Evaluationsprozess Empowerment und Capacity Building unterstützen kann, wenn er ermöglicht, dass die vorgesehenen Nutznießer des Programmes derart in die Bewertung einbezogen werden, dass sie bestimmte Fähigkeiten entwickeln und Ressourcen erhalten $[14,21,37,38]$. In den Interviews wurde deutlich, dass die Befragten partizipatorische Studienkonzepte vorziehen, die versuchen, die aktive Beteiligung der Gemeindemitglieder auch bei der wissenschaftlichen Komponente des Programms zu erreichen. Allerdings muss man berücksichtigen, dass die Gesundheitsförderer die Expertise für kooperative und partizipative Forschungsansätze möglicherweise erst erwerben müssen, was eine spezielle Weiterbildung notwendig macht [14]. Darüber hinaus wurde deutlich, dass man vorsichtig sein muss, von den Gemeindemitgliedern nicht zuviel zu verlangen, da einige von ihnen sicherlich mit forschungsbezogenen Aufgaben überfordert sind oder davon eher abgestoßen werden. Die Interviews ergaben, dass es nicht immer einfach und selbstverständlich ist, Studiendesigns und Datenerhebungsmethoden zu finden, die nicht einen paternalistischen Eindruck erwecken, und darüber hinaus nicht den Gemeindemitgliedern das Gefühl geben, dass das Messen wichtiger sei als das Handeln.
Obwohl die Befragten einhellig der Meinung waren, dass es wichtig sei, bei der Evaluation vor allem die Teilnehmer eines Programmes, d.h. die Gemeindemitglieder zu befragen, gab es unterschiedliche Vorstellungen dazu, wer die Gemeinde am besten repräsentieren kann: Aktiv einbezogene Gemeindeorganisationen, offizielle Gemeindevertreter, oder zufällig ausgewählte Bürger. Die Literatur unterstreicht ebenfalls, dass die Datenerhebung bei Menschen aus der gesamten Gemeinde geschehen soll [39], aber es fehlen belegte Aussagen darüber, wer denn als „repräsentativ" für die Gemeinde befragt werden soll. Die wenigen Studien, die zu diesem Thema durchgeführt wurden, haben jeweils unterschiedliche Datenquellen verwandt, z.B. indem willkürlich ausgewählte Gemeindemitglieder befragt wurden [4], Schlüsselpersonen [40], wichtige Teilnehmer [38], oder Projektkoordinatoren aus der Gemeinde [21].

Überwiegend wurde die Kombination von verschiedenen Datenquellen (sog. „source triangulation“) von den Interviewpartnern vorgezogen, was auch den Vorschlägen in der Literatur für die Evaluation von Gesundheitsförderung entspricht [12]. Die Literatur weist hier auch auf mögliche Probleme hin, die dadurch entstehen können, dass die Beurteilung von Empowerment sich aus Sicht der Gemeinde und aus Sicht der Projektleitern unterschiedlich ausfallen kann; dann muss geklärt werden, welcher Ansicht der Vorzug gegeben wird, z.B. bei der Festlegung zukünftiger Ressourcenallokation [41].

Die Interviewpartner waren größtenteils überzeugt vom Nutzen, den eine Bewertung von Empowerment hat, und hatten ermutigende Erfahrungen mit innovativen Evaluationsmethoden gemacht; dennoch gestanden sie ein, dass es durchaus schwierig sein kann, Evaluationsstudien zu entwickeln und durchzuführen. Einige der erwähnten Barrieren bezogen sich auf die zum Teil diffuse und schlecht operationalisierte Natur des Empowerment-Konzepts. Darüber hinaus zeigte sich, dass die Evaluation von Empowerment oft von Geldgebern und Entscheidungsträgern nicht unterstützt wird, da Empowerment gewöhnlich nicht als wichtiges Ergebnis einer gesundheitsfördernden Maßnahme gesehen wird, im Gegensatz zu Veränderungen in Gesundheitsverhalten und -zustand. Die Skepsis, die Entscheidungsträger dem Empowerment häufig entgegenbringen, wird unter Umständen noch dadurch verstärkt, dass qualitative Messmethoden, wie sie von den Gesundheitsförderern bevorzugt werden, wenig akzeptiert werden. Diese Eindrücke reflektieren Nutbeams Beobachtung, dass der quantitativen, experimentellen Forschung in Public Health ein höherer Status zukommt, und dass qualitative Methoden im Allgemeinen unterbewertet werden [12]. Trotzt dieser Schwierigkeiten, bevorzugte die Mehrheit der interviewten Experten qualitative Erhebungsverfahren für die Bewertung von Empowerment, weil sie geeigneter sind, die vielschichtigen, kontextbezogenen Facetten zu erheben, die sich im Zusammenhang mit Empowerment ergeben, und auch weil der Prozess der Datenerhebung und -auswertung selbst „empowernd“ wirken kann. Es ist interessant, dass die Befragten eher innovative und ungewöhnliche Evaluationsmethoden vorschlugen, z. B. Schauspiel, die Story Dialogue-Methode, eine Bürgerjury oder Photodokumentation, weniger die konventionellen und anerkannten qualitativen Verfahren wie Interviews der Fokusgruppen. Es lässt sich nur spekulieren, ob die Australier einen stärkeren Bezug zu diesen Methoden haben; immerhin wurde z. B. der Most Significant Change-Ansatz von Australiern entwickelt und hat in Australien bereits einige Verbreitung gefunden [42]. Die Literatur zeigt, dass Methoden wie Photodokumentation [43] oder der Story Dialogue-Ansatz, der aus Kanada stammt 
[44], v.a. von angelsächsischen Wissenschaftlern bzw. Gesundheitsförderern eingesetzt werden. Gleichzeitig sind die vorgeschlagenen Methoden auch als Ausdruck einer radikalen Umsetzung von Empowerment zu interpretieren.

Im Gegensatz zu den vorgeschlagenen Erhebungsmethoden befanden sich die in den Interviews genannten Indikatoren vorwiegend in Übereinstimmung mit den Variablen, wie sie die Literatur beschreibt. Es wurde allerdings deutlich, dass eine Reihe von Interviewpartnern die subjektiven Wahrnehmungen der Gemeindemitglieder, z.B. zu Fähigkeiten und Einfluss, nicht für ausreichend hielten, um bewerten zu können, ob ein umfassendes Empowerment tatsächlich erreicht werden konnte. Stattdessen schlugen sie vor, Wege zu finden, mit denen der tatsächliche Einfluss und die tatsächlichen Auswirkungen auf Entscheidungen und Ressourcenverfügung nachgezeichnet werden können, z.B. indem nachverfolgt wird, wie mit Vorschlägen von Gemeindemitgliedern im Laufe eines gemeindebasierten Programmes umgegangen wird, und ob und wie sie umgesetzt werden.

Die Studie gibt die Meinungen und Einschätzungen von Gesundheitsförderern eines australischen Bundesstaats wieder. Gleichwohl ist anzunehmen, dass die Aussagen, z.B. zu Indikatoren und Methoden, weitestgehend auf Deutschland übertragen werden können, da sie sich auf das Konzept des Empowerment selbst beziehen und wenig auf systemische bzw. landestypische Rahmenbedingungen. Ungewiss bleibt allerdings die kulturelle Akzeptanz der Evaluationsmethoden durch Mitglieder einer Gemeinde, in der bisher wenig mit dem Empowerment-Ansatz gearbeitet wurde. Dies mag insbesondere bei hochgradig partizipativen Verfahren wie Schauspiel oder Bürgerjurys gelten, bei denen ein hohes Engagement der Gemeindemitglieder gefordert ist, wie es in Deutschland vielleicht eher ungewöhnlich sein mag. Inwieweit die für eine Umsetzung von vielen der vorgeschlagenen Datenerhebungsmethoden notwendige Akzeptanz bei deutschen Projektleitern und Bürgern erreicht werden kann, muss zunächst eruiert werden. Die genannten Barrieren wie ein geringes Budget für Evaluation oder eingeschränkte methodische Ausbildung und Kompetenzen von vielen Gesundheitsförderern dürften ebenso auf deutsche Verhältnisse zutreffen $[45,46]$.

Es können also aus der Studie viele Erkenntnisse abgeleitet werden, die für die Evaluation von Empowerment im deutschen Kontext hilfreich sind. So konnte der Bedarf für eine breitere wissenschaftliche Diskussion des Ansatzes und die Arbeit an einer Verbesserung der Definition und Operationalisierung von Empowerment aufgezeigt werden. Ein gezieltes Bildungsangebot zur Messung und Bewertung von Empowerment bei Akteuren in der Gesundheitsförderung erscheint sinnvoll. Um praktische Erfahrungen mit der Evaluation von Empowerment in Deutschland sammeln zu können und die aus internationalen Studien übernommenen Evaluationskonzepte an die Besonderheiten im deutschen Raum anzupassen, müssten gezielt gesundheitsförderliche Programme gefördert werden, die Empowerment ausdrücklich als Element einbeziehen. Hier könnten dann exemplarische Evaluationen dieser Programme erfolgen, die die genannten methodischen Aspekte anwenden und gegebenenfalls weiterentwickeln. Unabhängig davon wäre es von großem Interesse, eine vergleichbare Erhebung auch bei deutschen Experten durchzuführen.

\section{Anmerkungen}

Die Studie wurde unterstützt durch die Deutsche Forschungsgemeinschaft, Forschungsstipendium LO 1186/1-1.

\section{Literatur}

1 World Health Organization. Declaration of Alma Ata (International conference on primary health care). WHO Europe, Copenhagen; 1978

2 World Health Organization. Ottawa Charter for Health Promotion. WHO Europe, Copenhagen; 1986

3 WHO Commission on the Social Determinants of Health. Achieving health equity: from root causes to fair outcomes. Geneva: World Health Organization, Commission on the Social Determinants of Health (CSDH); 2007

4 Israel BA, Checkoway B, Schulz A et al. Health education and community empowerment: conceptualizing and measuring perceptions of individual, organizational, and community control. Health Educ Q 1994; 21: 149-170

5 Wallerstein N. Powerlessness, empowerment, and health: implications for health promotion programs. Am J Health Promot 1992; 6: 197-205

6 Zimmerman MA. Psychological empowerment: issues and illustrations. Am J Community Psychol 1995; 23: 581-599

7 Rissel C. Empowerment: the holy grail of health promotion? Health Promot Int 1994; 9: 39-47

8 Laverack $G$, Wallerstein $N$. Measuring community empowerment: a fresh look at organizational domains. Health Promot Int 2001; 16: 179-185

9 Issel LM. Health Program Planning and Evaluation - A Practical, Systematic Approach for Community Health. Jones and Bartlett, London; 2004

10 Linnan L, Steckler A. Process evaluation for public health interventions and research - an overview. In: Steckler A, Linnan L, Hrsg. Process Evaluation for Public Health Interventions and Research. Jossey-Bass, San Francisco; 2004; 1-24

11 Potvin L, Richard L. Evaluating community health promotion programmes. WHO Reg Publ Eur Ser; 2001; 213-240

12 Nutbeam $D$. Evaluating health promotion - progress, problems and solutions. Health Promot Int 1998; 13: 27-44

13 Minkler M, Frantz S, Wechsler R. Social support and social action organizing in a "grey ghetto“: the tenderloin experience. 1982-83. Int Q Community Health Educ 2005; 25: 49-61

14 Wallerstein $N$. What is the evidence on effectiveness of empowerment to improve health? www.euro.who.int/Document/E88086.pdf abgerufen am 7.2.2007

15 Wallerstein N. Power between evaluator and community: research relationships within New Mexico's healthier communities. Soc Sci Med 1999; 49: 39-53

16 McMillan B, Florin P, Stevenson J et al. Empowerment praxis in community coalitions. Am J Community Psychol 1995; 23: 699-727

17 Rissel CE, Perry CL, Wagenaar AC et al. Empowerment, alcohol, 8th grade students and health promotion. J Alc Drug Educ 1996; 41: 105-119

18 Zimmerman MA, Rappaport J. Citizen participation, perceived control, and psychological empowerment. Am J Community Psychol 1988; 16 : 725-743

19 Fawcett SB, Paine-Andrews A, Francisco VT et al. Using empowerment theory in collaborative partnerships for community health and development. Am J Community Psychol 1995; 23: 677-697

20 Rissel C, Perry C, Finnegan J. Toward the assessment of psychological empowerment in health promotion: initial tests of validity and reliability. J R Soc Health 1996; 116: 211-218

21 Naylor PJ, Wharf-Higgins J, Blair L et al. Evaluating the participatory process in a community-based heart health project. Soc Sci Med 2002; 55: $1173-1187$

22 Zakus JD, Lysack CL. Revisiting community participation. Health Policy Plan 1998; 13: 1-12

23 Wen LM, Rissel C, Voukelatos A et al. Community involvement and selfrated health status: findings from a cross-sectional survey in Central Sydney. N S W Public Health Bull 2003; 14: 213-217

24 NSW Department of Health. A framework for building capacity to improve health. Sydney; 2001

25 NSW Department of Health. Strengthening health promotion in NSW - a map of the work and implications for workforce planning and development. Sydney; 2003 
26 McMeeking L, Kolpakow M von. Community and consumer participation in health. NSW Public Health Bull 2002; 13: 188

27 Baum F, Santich B, Craig B et al. Evaluation of a national health promotion program in South Australia. Aust N Z J Public Health 1996; 20: $41-49$

28 Clavarino AM, Janda $M$, Hughes $K L$ et al. The view from two sides: a qualitative study of community and medical perspectives on screening for colorectal cancer using FOBT. Prev Med 2004; 39: 482-490

29 Schofield MJ, Lynagh M, Mishra G. Evaluation of a Health Promoting Schools program to reduce smoking in Australian secondary schools. Health Educ Res 2003; 18: 678-692

30 Watson L, Small R, Brown S et al. Mounting a community-randomized trial: sample size, matching, selection, and randomization issues in PRISM. Control Clin Trials 2004; 25: 235-250

31 Harvey PW, Steele J, Bruggemann JN et al. The development and evaluation of lighten up, an Australian community-based weight management program. Am J Health Promot 1998; 13: 8-11

32 Rowley KG, Daniel M, Skinner K et al. Effectiveness of a communitydirected ,healthy lifestyle program in a remote Australian aboriginal community. Aust N Z J Public Health 2000; 24: 136-144

33 Loss J, Wise M. Concepts, benefits and limits of empowerment and participation in community-based health promotion practice - results of a qualitative study (eingereicht)

34 Patton MQ. Qualitative Evaluation and Research Methods. 2. Aufl. SAGE Publications, Newbury Park; 1990

35 Pope C, Ziebland S, Mays N. Qualitative research in health care. Analysing qualitative data. Bmj 2000; 320: 114-116

36 Mays $N$, Pope C. Qualitative research in health care. Assessing quality in qualitative research. Bmj 2000; 320: 50-52

37 Mayer SE. Building community capacity with evaluation activities that empower. In: Fetterman DE, Kaftarian SJ, Wandersman A, Hrsg. Empowerment Evaluation: Knowledge and tools for self-assessment and accountability. Thousand Oaks, London, New Delhi; 1996; 332-378
38 Fawcett SB, Paine-Andrews AL, Francisco VT et al. Empowering community health initiatives through evaluation. In: Fetterman DE, Kaftarian SJ, Wandersman A, Hrsg. Empowerment Evaluation: Knowledge and tools for self-assessment and accountability. Thousand Oaks, London, New Delhi; 1996; 161-187

39 Fawcett SB, Paine-Andrews A, Francisco VT et al Evaluating community initiatives for health and development. WHO Reg Publ Eur Ser; 2001; 241-270

40 Eng E, Parker E. Measuring community competence in the Mississippi Delta: the interface beween program evaluation and empowerment. Health Educ Q 1994; 21: 199-220

41 Dixon J, Sindall C. Applying the logics of change to the evaluation of community development in health promotion. Health Promot Int 1994; 9: 297-309

42 Davies $R$, Dart J. The ,Most Significant Change' technique: a guide to its use. http://www.mande.co.uk/docs/MSCGuide.pdf abgerufen am 24.4.2007

43 Wang C, Burris MA. Photovoice: concept, methodology, and use for participatory needs assessment. Health Educ Behav 1997; 24: 369-387

44 Labonte R, Feather J, Hills M. A story/dialogue method for health promotion knowledge development and evaluation. Health Educ Res 1999; 14: 39-50

45 Loss J, Eichhorn C, Reisig V et al. Qualitätsmanagement in der Gesundheitsförderung - Entwicklung eines multidimensionalen Qualitätssicherungsinstruments für eine landesweite Gesundheitsinitiative. Präv Gesundheitsförd 2007; 2: 199-206

46 Loss J. Editorial. Präv Gesundheitsförd 2008; 3: 53-55 